\title{
ON THE NEGATIVITY OF THE WALSH-KACZMARZ-RIESZ LOGARITHMIC KERNELS
}

\author{
György Gát ${ }^{1, a}$ and Gábor Lucskai ${ }^{1,2, *}$
}

\footnotetext{
${ }^{1}$ Institute of Mathematics, University of Debrecen, H-4002 Debrecen, Pf. 400, Hungary

${ }^{2}$ Institute of Mathematics and Informatics, University of Pécs, H-7624 Pécs, Ifjúság u. 6

Communicated by Margit Pap
}

Original Research Paper

Received: Sep 1, 2021 - Accepted: Oct 1, 2021

First published online: October 26, 2021

๑) 2021 The Author(s)

\section{ABSTRACT}

The main aim of this paper is to prove that the nonnegativity of the Riesz's logarithmic kernels with respect to the WalshKaczmarz system fails to hold.

\section{KEYWORDS}

Walsh-Kaczmarz, Riesz logarithmic kernel

\section{MATHEMATICS SUBJECT CLASSIFICATION (2020)}

Primary 42C10; Secondary

\section{INTRODUCTION}

The question of almost everywhere convergence is highly celebrated in the theory of Fourier series. It is quite well-known for both Walsh-Paley and trigonometric Fourier series, that the behavior of the logarihmic means

$$
\frac{1}{\log n} \sum_{k=1}^{n} \frac{S_{k}(f)}{k}
$$

is very nice. That is, for each function $f$ which is integrable on the unit interval, the logarithmic means converge to $f$ almost everywhere.

In [5] Hardy gave a necessary and sufficient condition for the convergence of a certain point of Riesz's logarithmic means of a function with respect to the trigonometric system. For some material concerning the trigonometric system see the book of Zygmund [2]. With respect to Walsh-PaleyRiesz logarithmic means see e.g. the paper of Gát and Goginava [4]. Behind of many results in the trigonometric case there is the fact that the Riesz's logarithmic kernel function is everywhere nonnegative. This very useful property of the kernel is given with the help of Abel transform and the fact that the Fejér kernels are everywhere nonnegative. On the other hand, it is quite simple to give Walsh-Paley-Fejér kernels which take negative values. That is, the method used in the

\footnotetext{
* Corresponding author. E-mail: glucskai@gamma.ttk.pte.hu

${ }^{a}$ gat.gyorgy@science.unideb.hu
} 
trigonometric case does not work as a proof of the nonnegativity. However, with a more difficult way the authors of this paper verified that Riesz's logarithmic kernels with respect the Walsh-Paley system take only nonnegative values. This result will be published elsewhere. The aim of this paper is to show that the Walsh-Paley and the Walsh-Kaczmarz system are different in this point of view. More precisely, we give a sequence of Walsh-Kaczmarz-Riesz logarithmic kernels such that all of them take negative values on some intervals. Next, we give some necessary preliminaries.

\section{PRELIMINARIES}

We follow the standard notions of dyadic analysis introduced by the mathematicians F. Schipp, P. Simon, W. R. Wade (see e.g. [1]) and others. Denote by $\mathbb{N}:=\{0,1, \ldots\}, \mathbb{P}:=\mathbb{N} \backslash\{0\}$, the set of natural numbers, the set of positive integers and $I:=[0,1)$ the unit interval. Denote by $\lambda(B)=|B|$ the Lebesgue measure of the set $B(B \subset I)$, with normalized Haar measure. Denote by $L^{p}(I)$ the usual Lebesgue spaces and $\|\cdot\|_{p}$ the corresponding norms $(1 \leq p \leq \infty)$. Let

$$
\mathcal{J}:=\left\{\left[\frac{p}{2^{n}}, \frac{p+1}{2^{n}}\right\}: p, n \in \mathbb{N}\right\}
$$

be the set of dyadic intervals and for given $x \in I$ and let $I_{n}(x)$ denote the interval $I_{n}(x) \in \mathcal{J}$ of length $2^{-n}$ which contains $x(n \in \mathbb{N})$. Also use the notation $I_{n}:=I_{n}(0)(n \in \mathbb{N})$. Let

$$
x=\sum_{n=0}^{\infty} x_{n} 2^{-(n+1)}
$$

the dyadic expansion of $x \in I$, where $x_{n}=0$ or 1 and if $x$ is a dyadic rational number $\left(x \in\left\{\frac{p}{2^{n}}\right.\right.$ : $p, n \in \mathbb{N}\}$ ) we choose the expansion which terminates in 0 's. The Rademacher functions are defined as:

$$
r_{n}(x)=(-1)^{x_{n}} \quad(x \in[0,1[, n \in \mathbb{N})
$$

Set the definition of the $n t h(n \in \mathbb{N})$ Walsh-Paley function at point $x \in I$ as:

$$
\omega_{n}(x):=\prod_{j=0}^{|n|}\left(r_{j}(x)\right)^{n_{j}}=(-1)^{\sum_{j=0}^{|n|} n_{j} x_{j}} .
$$

We defined the set of Walsh-Paley system: $\omega:=\left(\omega_{n}, n \in \mathbb{N}\right)$.

The $n$th $(n \in \mathbb{P})$ Walsh-Kaczmarz function at point $x \in I$ is defined as ([6], [1]):

$$
\kappa_{n}(x):=r_{|n|}(x) \prod_{k=0}^{|n|-1}\left(\eta_{n \mid-1-k}(x)\right)^{n_{k}}=r_{|n|}(x)(-1)^{\sum_{k=0}^{|n|-1} n_{k} x_{|n|-1-k}}
$$

where $\mathbb{P} \ni n=\sum_{n=0}^{\infty} n_{j} 2^{j}\left(n_{j} \in\{0,1\}(j \in \mathbb{N})\right)$, and $\kappa_{0}=1$. The Walsh-Kaczmarz system is denoted as $\kappa:=\left\{\kappa_{n}, n \in \mathbb{N}\right\}$.

By means of the transformation $\tau_{A}: I \rightarrow I,(A \in \mathbb{N})$

$$
\left(x_{0}, x_{1}, \ldots, x_{A-1} x_{A}, x_{A+1}, \ldots\right) \rightarrow\left(x_{A-1}, x_{A-2}, \ldots, x_{1}, x_{0}, x_{A}, x_{A+1}, \ldots\right),
$$

which is clearly measure-preserving and such that $\tau_{A}\left(\tau_{A}(x)\right)=x$ we will be able to discuss the values of Walsh-Kaczmarz functions and kernels. That is, we have (see also [6]) the following important equality:

$$
\kappa_{n}(x)=\eta_{n \mid}(x) \omega_{n}\left(\tau_{n}(x)\right) \quad(n \in \mathbb{N}) .
$$

It is known [1] that the system $\left(\omega_{n}, n \in \mathbb{N}\right)$ is the character system of $(I,+)$, where the group operation + is the so-called dyadic or logical addition on $I$. That is, for any $x, y \in I$

$$
x+y:=\sum_{n=0}^{\infty}\left|x_{n}-y_{n}\right| 2^{-(n+1)} .
$$

Let $\alpha$ be either $\kappa$ or $\omega$. Denote by

$$
D_{n}^{\alpha}:=\sum_{k=0}^{n-1} \alpha_{k}, \quad K_{n}^{\alpha}:=\frac{1}{n} \sum_{k=0}^{n-1} D_{k}^{\alpha}, \quad R_{n}^{\alpha}:=\frac{1}{\log n} \sum_{k=1}^{n-1} \frac{D_{k}^{\alpha}}{k} \quad D_{0}^{\alpha}, K_{0}^{\alpha}:=0
$$


the Dirichlet, the Fejér or $(C, 1)$ kernels and Walsh-logarithmic means, respectively. If in the notation $\alpha$ is missing, then we mean the Walsh-Paley ordering. Moreover: ([1])

$$
\begin{aligned}
& D_{2^{n}}^{\alpha}(x)= \begin{cases}2^{n}, & x \in I_{n}, \\
0, & x \notin I_{n},\end{cases} \\
& D_{n}(x)=\omega_{n}(x) \sum_{i=0}^{\infty} n_{i} r_{i} D_{2^{i}}(x) .
\end{aligned}
$$

It is also known that the Fourier coefficient, the $n$th partial sum of Fourier series and the Fejér or $(C, 1)$ means of $f$,

$$
\begin{aligned}
\hat{f}^{\alpha}(n) & :=\int_{I} f(x) \alpha_{n}(x) d \lambda(x) \quad(n \in \mathbb{N}), \\
S_{n}^{\alpha} f(y) & :=\sum_{k=0}^{n-1} \hat{f^{\alpha}}(k) \alpha_{k}(y)=\int_{I} f(x+y) D_{n}^{\alpha}(x) d \lambda(x) \quad\left(n \in \mathbb{P}, S_{0}^{\alpha} f=0\right), \\
\sigma_{n}^{\alpha} f(y) & :=\frac{1}{n} \sum_{k=0}^{n-1} S_{k}^{\alpha} f(y)=\int_{I} f(x) K_{n}^{\alpha}(y+x) d \lambda(x) \quad\left(n \in \mathbb{P}, \sigma_{0}^{\alpha} f=0, y \in I\right) .
\end{aligned}
$$

\subsection{The key observation}

In the sequel we will need the following lemma.

LEMMA 2.1. For $j, n \in \mathbb{N}, j<2^{n}$ we have

$$
D_{2^{n}+j}^{\kappa}(x)=D_{2^{n}}(x)+r_{n}(x) D_{j}^{\omega}\left(\tau_{n} x\right) .
$$

LEMMA 2.2 (Young-inequality). [8] Let $n \in \mathbb{P}$. Then

$$
\frac{1}{2(n+1)}<\sum_{k=1}^{n} \frac{1}{k}-\log (n)-\gamma<\frac{1}{2 n},
$$

where $\gamma=\lim _{n \rightarrow \infty}\left(\sum_{k=1}^{n} \frac{1}{k}-\log (n)\right)$.

COROLLARY 2.3. Let $n, N \in \mathbb{N}$ and $N>n$. Then

$$
0<\sum_{k=n}^{N-1} \frac{1}{k}-\log \left(\frac{N}{n}\right)<\frac{1}{n} .
$$

The main aim of this paper is to prove the following negativity result concerning the Riesz's logarithmic kernels with respect to the Walsh-Kaczmarz system.

THEOREM 2.4. Let be $A=2^{n}+2^{n-1}$ and $x=\frac{1}{2}+\frac{1}{4}+\frac{1}{2^{n+1}}$, where $n \in \mathbb{N}$. Then

$$
\log (A) R_{A}^{\kappa}(x) \leq-0.02 \cdot 2^{n},
$$

if $n \geq 11$.

\section{PROOFS}

Proof of Lemma 2.1. Use the formula of Skvortsov for Walsh-Kaczmarz-Dirichlet functions in [6].

$$
D_{2^{n}+j}^{\kappa}(x)=\sum_{k=0}^{2^{n}-1} \kappa_{k}(x)+\sum_{k=0}^{j-1} \kappa_{2^{n}+k}(x)=D_{2^{n}}(x)+r_{n}(x) D_{j}^{\omega}\left(\tau_{n} x\right) .
$$

This completes the proof of Lemma 2.1.

Next, we turn our attention to the proof of the main result. That is, to Theorem 2.4. 
Proof.

$$
\begin{aligned}
\log (A) R_{A}^{\kappa}(x) & =\sum_{j=1}^{2^{n}+2^{n-1}-1} \frac{D_{j}^{\kappa}(x)}{j}=\sum_{j=1}^{2^{n}-1} \frac{D_{j}^{\kappa}(x)}{j}+\sum_{j=2^{n}}^{2^{n}+2^{n-1}-1} \frac{D_{j}^{\kappa}(x)}{j} \\
& =\sum_{i=0}^{n-1} \sum_{l=2^{i}}^{2^{i+1}-1} \frac{D_{l}^{\kappa}(x)}{l}+\sum_{j=2^{n}}^{2^{n}+2^{n-1}-1} \frac{D_{j}^{\kappa}(x)}{j} \\
& =\sum_{i=0}^{n-1} \sum_{t=0}^{2^{i}-1} \frac{D_{2^{i}+t}^{\kappa}(x)}{2^{i}+t}+\sum_{t=0}^{2^{n-1}-1} \frac{D_{2^{n}+t}^{\kappa}(x)}{2^{n}+t}=(1)+(2) .
\end{aligned}
$$

We start with estimation of (2): Notice that $r_{n}(x)=-1, D_{2^{n}}(x)=0$ and

$$
\tau_{n}(x)=(0,0, \ldots, 0,1,1,1,0, \ldots)=\left(y_{0}, y_{1}, \ldots, y_{n-3}, y_{n-2}, y_{n-1}, y_{n}, y_{n+1}, \ldots\right) .
$$

Furthermore,

$$
D_{l}\left(\tau_{n}(x)\right)=l, \quad \text { for } l=0,1,2, \ldots, 2^{n-2}-1 .
$$

Let $l \in\left\{2^{n-2}, \ldots, 2^{n-1}-1\right\}$. Then $l=2^{n-2}+b$, where $b \in\left\{0,1, \ldots, 2^{n-2}-1\right\}$. By this we have

$$
D_{l}\left(\tau_{n}\right)=D_{2^{n-2}+b}\left(\tau_{n}\right)=D_{2^{n-2}}\left(\tau_{n}\right)+r_{n-2}\left(\tau_{n}(x)\right) D_{b}\left(\tau_{n}\right)=2^{n-2}+(-1) \cdot b .
$$

Then

$$
\begin{aligned}
(2) & =\sum_{t=0}^{2^{n-1}-1} \frac{D_{2^{n}+t}^{K}(x)}{2^{n}+t}=\sum_{t=0}^{2^{n-1}-1} \frac{D_{2^{n}}(x)+r_{n}(x) D_{t}\left(\tau_{n}(x)\right)}{2^{n}+t} \\
& =\sum_{t=0}^{2^{n-1}-1} \frac{-D_{t}\left(\tau_{n}(x)\right)}{2^{n}+t} \\
& =\sum_{t=0}^{2^{n-2}-1} \frac{-D_{t}\left(\tau_{n}(x)\right)}{2^{n}+t}+\sum_{t=2^{n-2}}^{2^{n-1}-1} \frac{-D_{t}\left(\tau_{n}(x)\right)}{2^{n}+t} \\
& =\sum_{t=0}^{2^{n-2}-1} \frac{-t}{2^{n}+t}-\sum_{b=0}^{2^{n-2}-1} \frac{2^{n-2}-b}{2^{n}+2^{n-2}+b} \\
& =\sum_{t=2^{n}+2^{n-2}-1} \frac{2^{n}-t}{t}-\sum_{t=5 \cdot 2^{n-2}}^{6 \cdot 2^{n-2}-1} \frac{2^{n-2}+5 \cdot 2^{n-2}-t}{t} \\
& =2^{n} \sum_{t=4 \cdot 2^{n-2}}^{5 \cdot 2^{n-2}-1} \frac{1}{t}-\sum_{t=4 \cdot 2^{n-2}}^{5 \cdot 2^{n-2}-1} 1-6 \cdot 2^{n-2} \sum_{t=5 \cdot 2^{n-2}}^{6 \cdot 2} \frac{1}{t}+\sum_{t=5 \cdot 2^{n-2}}^{6 \cdot 2^{n-2}-1} 1 \\
& <2^{n}\left(\log \left(\frac{5}{4}\right)+\frac{1}{4 \cdot 2^{n-2}}-\frac{6}{4} \cdot \log \left(\frac{6}{5}\right)\right) .
\end{aligned}
$$

That is, it is left to give an estimation for (1). We apply Lemma 2.1. That is,

$$
\sum_{i=0}^{n-1} \sum_{t=0}^{2^{i}-1} \frac{D_{2^{i}+t}^{\kappa}(x)}{2^{i}+t}=\frac{D_{1}^{\kappa}(x)}{1}+\frac{D_{2}^{\kappa}(x)}{2}+\frac{D_{3}^{\kappa}(x)}{3}+\sum_{i=2}^{n-1} \sum_{t=0}^{2^{i}-1} \frac{D_{2^{i}}(x)+r_{i}(x) D_{t}\left(\tau_{i}(x)\right)}{2^{i}+t} .
$$


We have $D_{2^{i}}^{\alpha}(x)=0$, for $i=1,2, \ldots n$. For $2 \leq i<n$ it holds $r_{i}(x)=1$. Furthermore $r_{0}(x)=\kappa_{1}(x)=$ $\omega_{1}(x)$ and $\kappa_{2}(x)=\omega_{2}(x)=r_{1}(x)$. Now, return to the investigation of (1).

$$
\begin{aligned}
& \sum_{i=0}^{n-1} \sum_{t=0}^{2^{i}-1} \frac{D_{2^{i}+t}^{\kappa}(x)}{2^{i}+t}=1+0-\frac{1}{3}+\sum_{i=2}^{n-1} \sum_{t=0}^{2^{i}-1} \frac{D_{t}\left(\tau_{i}(x)\right)}{2^{i}+t} \\
& \quad=\frac{2}{3}+\sum_{i=2}^{n-1}\left(\sum_{t=0}^{2^{i-2}-1} \frac{D_{t}\left(\tau_{i}(x)\right)}{2^{i}+t}+\sum_{t=2^{i-2}}^{2^{i-1}-1} \frac{D_{t}\left(\tau_{i}(x)\right)}{2^{i}+t}+\sum_{t=2^{i-1}}^{2^{i-1}+2^{i-2}-1} \frac{D_{t}\left(\tau_{i}(x)\right)}{2^{i}+t}+\sum_{t=2^{i-1}+2^{i-2}-1}^{2^{i}-1} \frac{D_{t}\left(\tau_{i}(x)\right)}{2^{i}+t}\right) \\
& =: \frac{2}{3}+\sum_{i=2}^{n-1}((1.1)+(1.2)+(1.3)+(1.4)) .
\end{aligned}
$$

In the sequel recall that $\tau_{i}(x)=\frac{1}{2^{i-1}}+\frac{1}{2^{i}}+\frac{1}{2^{n+1}}=: y_{i-2}^{i} e_{i-2}+y_{i-1}^{i} e_{i-1}+y_{n}^{i} e_{n}$, where $e_{k}: 1 / 2^{k+1}$. That is, note that the only coordinates (with not greater index than $n$ ) of $y^{i}=\tau_{i}(x)$ different from zero are $y_{i-2}, y_{i-1}$ and $y_{n}$ for any $i<n$. Now, turn our attention to the investigation of (1.1).

$$
\begin{aligned}
(1.1) & =\sum_{t=0}^{2^{i-2}-1} \frac{D_{t}\left(\tau_{i}(x)\right)}{2^{i}+t}=\sum_{t=0}^{2^{i-2}-1} \frac{t}{2^{i}+t}= \\
& =\sum_{l=2^{i}}^{2^{i}+2^{i-2}-1} \frac{l-2^{i}}{l}=\sum_{l=4 \cdot 2^{i-2}}^{5 \cdot 2^{i-2}-1} 1-2^{i} \sum_{l=4 \cdot 2^{i-2}}^{5 \cdot 2^{i-2}-1} \frac{1}{l} \\
& <2^{i}\left(\frac{1}{4}-\log \left(\frac{5}{4}\right)\right) .
\end{aligned}
$$

Meanwhile for (1.2) we have

$$
\begin{aligned}
(1.2) & =\sum_{t=2^{i-2}}^{2^{i-1}-1} \frac{D_{t}\left(\tau_{i}(x)\right)}{2^{i}+t}=\sum_{l=0}^{2^{i-2}-1} \frac{D_{2^{i-2}+l}\left(\tau_{i}(x)\right)}{2^{i}+2^{i-2}+l} \\
& =\sum_{l=0}^{2^{i-2}-1} \frac{D_{2^{i-2}}\left(\tau_{i}(x)\right)+r_{i-2}\left(\tau_{i}(x)\right) D_{l}\left(\tau_{i}(x)\right)}{2^{i}+2^{i-2}+l} \\
& =\sum_{l=0}^{2^{i-2}-1} \frac{2^{i-2}-l}{2^{i}+2^{i-2}+l}=\sum_{t=5 \cdot 2^{i-2}}^{6 \cdot 2^{i-2}-1} \frac{6 \cdot 2^{i-2}-t}{t} \\
& =6 \cdot 2^{i-2} \sum_{t=5 \cdot 2^{i-2}}^{6 \cdot 2^{i-2}-1} \frac{1}{t \cdot 2^{i-2}-1} \sum_{t=5 \cdot 2^{i-2}} 1 \\
& <2^{i}\left(\frac{6}{4} \log \left(\frac{6}{5}\right)+\frac{6}{4 \cdot 5 \cdot 2^{i-2}}-\frac{1}{4}\right)
\end{aligned}
$$

Similarly, we investigate the term (1.3) as follows:

$$
\begin{aligned}
(1.3) & =\sum_{t=2^{i-1}}^{2^{i-1}+2^{i-2}-1} \frac{D_{t}\left(\tau_{i}(x)\right)}{2^{i}+t}=\sum_{l=0}^{2^{i-2}-1} \frac{D_{2^{i-1}+l}\left(\tau_{i}(x)\right)}{2^{i}+2^{i-1}+l} \\
& =\sum_{l=0}^{2^{i-2}-1} \frac{D_{2^{i-1}}\left(\tau_{i}(x)\right)+r_{i-1}\left(\tau_{i}(x)\right) D_{l}\left(\tau_{i}(x)\right)}{2^{i}+2^{i-1}+l} \\
& =\sum_{l=0}^{2^{i-2}-1} \frac{-l}{6 \cdot 2^{i-2}+l}=\sum_{z=6 \cdot 2^{i-2}}^{7 \cdot 2^{i-2}-1} \frac{6 \cdot 2^{i-2}-z}{z} \\
& <2^{i} \cdot\left(\frac{6}{4} \cdot \log \left(\frac{7}{6}\right)+\frac{6}{6 \cdot 4 \cdot 2^{i-2}}-\frac{1}{4}\right) .
\end{aligned}
$$


At last, we have to discuss (1.4)

$$
\begin{aligned}
& (1.4)=\sum_{t=2^{i-1}+2^{i-2}}^{2^{i}-1} \frac{D_{t}\left(\tau_{i}(x)\right)}{2^{i}+t}=\sum_{l=2^{i-2}}^{2^{i-1}-1} \frac{D_{2^{i-1}+l}\left(\tau_{i}(x)\right)}{2^{i}+2^{i-1}+l} \\
& =\sum_{l=2^{i-2}}^{2^{i-1}-1} \frac{D_{2^{i-1}}\left(\tau_{i}(x)\right)+r_{i-1}\left(\tau_{i}(x)\right) D_{l}\left(\tau_{i}(x)\right)}{2^{i}+2^{i-1}+l} \\
& =\sum_{l=2^{i-2}}^{2^{i-1}-1} \frac{-D_{l}\left(\tau_{i}(x)\right)}{2^{i}+2^{i-1}+l}=\sum_{j=0}^{2^{i-2}-1} \frac{-D_{2^{i-2}+j}\left(\tau_{i}(x)\right)}{2^{i}+2^{i-1}+2^{i-2}+j} \\
& =\sum_{j=0}^{2^{i-2}-1} \frac{-D_{2^{i-2}}\left(\tau_{i}(x)\right)-r_{i-2}\left(\tau_{i}(x)\right) D_{j}\left(\tau_{i}(x)\right)}{2^{i}+2^{i-1}+2^{i-2}+j} \\
& =\sum_{j=0}^{2^{i-2}-1} \frac{-2^{i-2}+j}{7 \cdot 2^{i-2}+j}=\sum_{j=7 \cdot 2^{i-2}}^{8 \cdot 2^{i-2}-1} \frac{-8 \cdot 2^{i-2}+j}{j} \\
& =-8 \cdot 2 \sum_{j=7 \cdot 2^{i-2}}^{8 \cdot 2} \frac{2^{i-2}-1}{j}+\sum_{j=7 \cdot 2^{i-2}}^{8 \cdot 2^{i-2}-1} 1 \\
& <2^{i}\left(-\frac{8}{4} \log \left(\frac{8}{7}\right)+\frac{1}{4}\right) \text {. }
\end{aligned}
$$

These assumptions above lead us to the estimation of (1) wanted as follows:

$$
\begin{aligned}
\sum_{i=0}^{n-1} \sum_{t=0}^{2^{i}-1} \frac{D_{2^{i}+t}^{\kappa}(x)}{2^{i}+t}= & \frac{2}{3}+\sum_{i=2}^{n-1}((1.1)+(1.2)+(1.3)+(1.4)) \\
< & \frac{2}{3}+\sum_{i=2}^{n-1} 2^{i}\left(\frac{1}{4}-\log \left(\frac{5}{4}\right)+\frac{6}{4} \log \left(\frac{6}{5}\right)+\frac{6}{4 \cdot 5 \cdot 2^{i-2}}-\frac{1}{4}\right. \\
& \left.+\frac{6}{4} \cdot \log \left(\frac{7}{6}\right)+\frac{6}{6 \cdot 4 \cdot 2^{i-2}}-\frac{1}{4}-\frac{8}{4} \log \left(\frac{8}{7}\right)+\frac{1}{4}\right) \\
\leq & \frac{2}{3}+\sum_{i=2}^{n-1}\left[2^{i}\left(-\log \left(\frac{5}{4}\right)+\frac{6}{4} \log \left(\frac{6}{5}\right)+\frac{6}{4} \cdot \log \left(\frac{7}{6}\right)-\frac{8}{4} \log \left(\frac{8}{7}\right)\right)+\frac{11}{5}\right] \\
= & 2^{n} \cdot\left(-\log \left(\frac{5}{4}\right)+\frac{6}{4} \log \left(\frac{6}{5}\right)+\frac{6}{4} \cdot \log \left(\frac{7}{6}\right)-\frac{8}{4} \log \left(\frac{8}{7}\right)\right)+\frac{11}{5}(n-2)+\frac{2}{3}
\end{aligned}
$$

Finally, we have the inequality

$$
\begin{aligned}
\log (A) R_{A}^{\kappa}(x) & =(1)+(2)=\sum_{i=0}^{n-1} \sum_{t=0}^{2^{i}-1} \frac{D_{2^{i}+t}^{\kappa}(x)}{2^{i}+t}+\sum_{t=0}^{2^{n-1}-1} \frac{D_{2^{n}+t}^{\kappa}(x)}{2^{n}+t} \\
& <2^{n} \cdot 0.01451+\frac{11}{5}(n-2)+\frac{2}{3}-2^{n} \cdot 0.050337+1 \\
& =-0.035827 \cdot 2^{n}+2.2 \cdot(n-2)+\frac{5}{3} \leq-0.02 \cdot 2^{n},
\end{aligned}
$$

if $n \geq 11$. This completes the proof of Theorem 2.4.

\section{ACKNOWLEDGEMENTS}

Research is supported by the Hungarian National Foundation for Scientific Research (OTKA), grant no. K111651 and by project EFOP-3.6.2-16-2017-00015 supported by the European Union, co-financed by the European Social Fund. 


\section{REFERENCES}

[1] Schipp, F., WAde, W. R., Simon, P., and Pál, J. Walsh series: an introduction to dyadic harmonic analysis. Adam Hilger, Bristol and New York, 1990.

[2] Zygmund, A. Trigonometric Series.. University Press, Cambridge, 1959 (English).

[3] GÁt, G. On $(C, 1)$ summability of integrable functions with respect to the Walsh-Kaczmarz system. Studia Math 130, 2 (1998), 135-148.

[4] GÁt, G., and Goginava, U. Uniform and L-convergence of logarithmic means of Walsh-Fourier series, Acta Mathematica Sinica 22, 2 (2006), 497-506.

[5] Hardy, G. H. The summability of a Fourier series by logarithmic means. The Quarterly fournal of Mathematics 1, (1931), 107-112.

[6] Skvorcov, V. A. On Fourier series with respect to the Walsh-Kaczmarz system. Analysis Math. 7 (1981), 141-150.

[7] Skvorcov, V. A. Convergence in $L^{1}$ of Fourier series with respect to the Walsh-Kaczmarz system. Vestnik Mosk. Univ. Ser. Mat. Meh. 6 (1981), 3-6.

[8] Young, R. M. Euler's Constant. The Mathematical Gazette 75, 472 (Jun. 1991), 187-190.

Open Access statement. This is an open-access article distributed under the terms of the Creative Commons Attribution 4.0 International License (https://creativecommons.org/licenses/by/4.0/), which permits unrestricted use, distribution, and reproduction in any medium, provided the original author and source are credited, a link to the CC License is provided, and changes - if any - are indicated. (SID_1) 